\title{
Labyrinthe
}

$6 \mid 2000$

Numéro 6

\section{Les sens des savoirs}

Yves Michaud

\section{(2) OpenEdition}

Journals

Édition électronique

URL : http://journals.openedition.org/labyrinthe/439

DOI : $10.4000 /$ labyrinthe.439

ISSN : 1950-6031

Éditeur

Hermann

Édition imprimée

Date de publication : 1 juin 2000

Pagination : 9-30

Référence électronique

Yves Michaud, «Les sens des savoirs », Labyrinthe [En ligne], 6 | 2000, mis en ligne le 18 mars 2005, consulté le 19 avril 2019. URL : http://journals.openedition.org/labyrinthe/439;DOI : 10.4000/

labyrinthe.439

Ce document a été généré automatiquement le 19 avril 2019

Propriété intellectuelle 


\title{
Les sens des savoirs
}

\author{
Yves Michaud
}

1 Labyrinthe - Vous êtes le concepteur de l'Université de Tous Les Savoirs (UTLS), qui, dans le cadre de la Mission 2000 en France, propose chaque jour de l'année 2000 une conférence au Conservatoire National des Arts et Métiers (CNAM) ${ }^{1}$. Quelle est l'ambition de ce projet ? S'agit-il de constituer une encyclopédie vivante et de renouer ainsi avec la tradition de l'Encyclopédie, qu'évoque le lieu que vous avez choisi', fondé par l'abbé Grégoire au XVIII siècle?

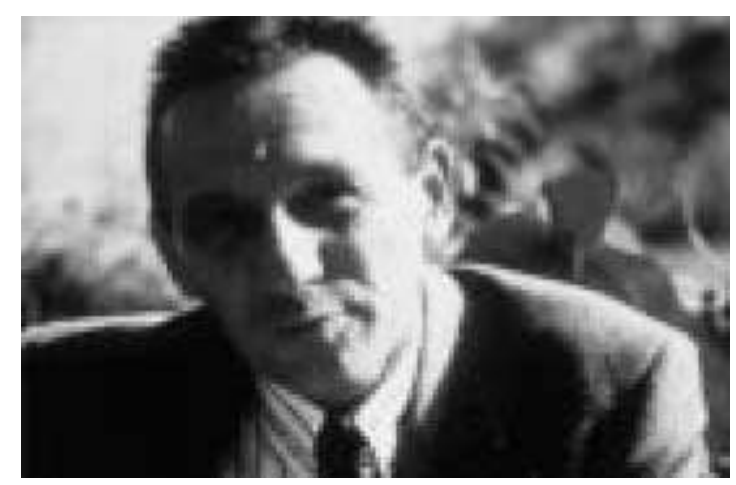

Yves Michaud

2 Yves Michaud - La vocation de l'UTLS n'est pas encyclopédique au sens où les encyclopédies sont des réservoirs de savoirs finalement assez statiques, qui supposent un parcours très ordonné et relativement figé de la connaissance ${ }^{3}$. Il faut plutôt voir, dans l'Université de Tous Les Savoirs, une volonté de parcourir le savoir, le plus complètement possible, certes, mais de manière orientée, prospective, avec des prises de risques calculées, dans l'ignorance délibérée de certains sujets ${ }^{4}$, ou bien, au contraire dans l'intérêt prononcé pour d'autres. L'ambition - à la fois modeste et audacieuse - de ce projet est d'apporter des éclairages sur les questions importantes et les évolutions récentes dans le champ du savoir ${ }^{5}$ et des techniques, qui, selon nos paris, se révéleront des enjeux cruciaux à moyen terme, dans cinq ans ou dix ans ${ }^{6}$. 
3 L. - Le programme de l'UTLS' est divisé en trois grands blocs : l'homme (la vie, l'évolution, les conditions chimiques et biochimiques mais aussi les conditions sociales et culturelles de l'existence humaine) ; les sciences exactes (mathématiques, géographie, chimie...) ; et l'enseignement, les technologies et les enjeux plus concrets (aussi bien géopolitiques, que culturels et éthiques) des années qui viennent. À première vue, les sciences humaines sont le parent pauvre de votre programme ${ }^{8}$, ce qui est d'autant plus surprenant, voire déconcertant, de la part du philosophe que vous êtes également. Pouvez-vous nous expliquer votre démarche?

4 Y.M. - Il était prévu dès l'origine qu'un programme de conférences soit consacré pour deux tiers aux sciences dites " exactes » et pour un tiers aux sciences humaines'. En réalité, les sciences humaines sont davantage représentées, même si elles ne sont pas toujours signalées en tant que telles, et ne font pas l'objet, à elles seules, théoriquement, d'autres conférences. Prenons l'exemple du droit : il n'est à proprement parler traité que dans trois conférences ${ }^{10}$, mais les questions juridiques sont néanmoins abordées à travers de nombreux autres sujets d'interventions ${ }^{11}$. Le droit est donc très présent, transversalement.

5 Notre souci permanent d'éviter le plus possible une approche académique nous interdisait le cloisonnement des disciplines et les classifications (et donc le cheminement du savoir) dans l'ordre des rubriques : sociologie, psychologie, droit, etc. Ce qui explique la non-visibilité immédiate de cette présence pourtant incontestable des sciences humaines dans le programme de l'UTLS. Il existe en effet un découpage académique des savoirs, de même que des découpages disciplinaires ont cours par exemple au CNRS ou dans certaines sections du Comité National des Universités $(\mathrm{CNU})^{12}$. Mais il me semblait difficile et relativement ennuyeux d'élaborer une grande fresque persuasive en reprenant des épisodes académiques ! J'ai ainsi dû inventer, créer, pour l'occasion de l'UTLS, une structure narrative qui devait être lisible à la fois par les conférenciers et par le public : un scénario n'entrant donc absolument pas dans une structure disciplinaire reprenant l'ordre de l'Académie des sciences, puis de l'Académie des Belles Lettres, de l'Académie des Beaux-Arts ${ }^{13}$, etc.

6 Quant à la place des sciences humaines dans le champ du savoir, je pense qu'elles sont la plupart du temps travaillées d'un point de vue pluridisciplinaire et en rapport avec des problématiques concrètes. Voilà pour l'éloge. En revanche, elles souffrent à mon avis d'un retard conceptuel et d'une théorisation déficiente, même si cela n'est pas vrai pour toutes les sciences humaines. Ainsi, je n'entrevois toujours pas de percée des sciences sociales. En sociologie ${ }^{14}$, peu d'évolutions se produisent, et en psychologie ${ }^{15}$ rien ne change.

7 L. - Vous avez donc organisé le programme des conférences comme un scénario très pluridisciplinaire. Vous avez vous-même une formation de cet ordre puisque vous êtes professeur de philosophie mais avez également étudié la sociologie, les sciences, l'histoire de l'art ${ }^{16}$, et continuez à vous intéresser à des domaines très variés. Quelle est votre point de vue sur la pluridisciplinarité ?

8 Y.M. - Je suis depuis toujours un fervent partisan de la pluridisciplinarité. Pour moi, ce type d'approche est naturel ${ }^{17}$. Les appréhensions pluridisciplinaires, transdisciplinaires ou interdisciplinaires ne visent pas à tout mélanger mais sont fondamentales car elles sont une manière de multiplier les éclairages sur un certain nombre de phénomènes. Les concepts sont des sortes d'« outils » intellectuels qu'on utilise pour " bricoler », en 
philosophi ${ }^{18}$, ainsi que dans les autres disciplines. J'aime cette idée de "boite à outils » que développe Wittgenstein ${ }^{19}$.

Mais je suis bientôt parvenu à la fin de ma carrière, et si j'ai abondamment entendu parler toute ma vie de la nécessité de la pluridisciplinarité, je ne l'ai jamais vue mise en œuvre véritablement ! À cet égard, l'histoire ne me parait pas être dans une meilleure situation que la philosophie : elle reste très académique et semble avoir un peu oublié ses démarches et ses méthodes. Jacques Le Goff déplore lui-même ${ }^{20}$ cette tendance actuelle à " raconter des histoires " et à refaire des lectures en ne revenant jamais aux documents, aux archives, et tout simplement à la recherche. On a toujours tendance à triturer un matériau assez limité, de seconde main, et corrélativement, la conceptualisation laisse fortement à désirer. Or, je crois que toute discipline a besoin de ces deux éléments fondamentaux que sont le matériau et le concept $t^{21}$. La même situation existe en histoire de l'art, qui souffre d'un réel manque d'études de fond ${ }^{22}$.

Pourtant, très concrètement, la pluridisciplinarité existe et est parfois élaborée par de jeunes chercheurs qui l'estiment à juste titre nécessaire. Je peux ainsi citer l'exemple d'une brillante maîtrise réalisée par une étudiante en histoire, qui a réfléchi sur la notion de " grand homme » en Allemagne à la fin du XIXe siècle, sous l'angle double de l'histoire et de la philosophie. Il s'agit d'un cas typique où une approche pluridisciplinaire s'impose : le " grand homme " est en effet un concept philosophique très courant à partir des années 1830-1840, mais aussi une notion fondamentale dans l'histoire de la pensée du XIXe siècle. Il est très intéressant d'analyser comment la littérature politique et le journalisme politique ont pu appréhender cette notion, qui implique à la fois une approche conceptuelle et une recherche historique de base.

11 Mais la France n'a malheureusement pas une culture, une tradition pluridisciplinaire, ce qui se ressent très fortement au niveau de l'Institution universitaire. La pluridisciplinarité a bien ses sanctuaires, comme l'École des Hautes Études en Sciences Sociales (EHESS ${ }^{23}$ ), Sciences-Po (IEP $\left.{ }^{24}\right)$ dans une certaine mesure, ou encore le Collège International de Philosophie $\left(\mathrm{CIP}^{25}\right)$. Mais, presque toujours, les institutions à vocation transdisciplinaire qui se créent en France participent progressivement d'une forme d'académisme d'un nouveau genre. Mon militantisme sur ce sujet me place, vis-à-vis de mes collègues, dans une position d'outsider ${ }^{26}$ total.

L. - Vous formulez en effet des critiques très sévères sur l'Université...

13 Y.M. - Je considère que l'Université est totalement rigide et immobiliste, en tout cas en sciences humaines car je ne me prononcerai pas sur les sciences exactes qui relèvent d'un domaine où je ne prétends pas juger. La cause de cette situation calamiteuse est purement sociologique : le corporatisme ${ }^{27}$ et la gestion des carrières, par le CNRS et par les sections du CNU compétentes en matière de recrutements, d'habilitations et de promotions. Les professeurs défendent leur corporation pour mieux la reproduire : l'université est le lieu de la reproduction ${ }^{28}$ par excellence.

Reprenons l'exemple de la pluridisciplinarité : cela fait quarante ans qu'on parle de l'inscrire dans les faits ! En 1968, la réforme d'Edgar Faure était destinée à favoriser la " multidisciplinarité » et proposait d'instaurer des doubles DEUG pluridisciplinaires. Or, tout l'effort auquel j'ai assisté de la part de mes collègues, pendant les années 70, a été de lutter pour rétablir les DEUG mono-disciplinaires ! Deuxième acte $:$ la réforme Bayrou ${ }^{30} \mathrm{a}$ 
permis d'introduire des UV de culture générale en $1^{\text {er }}$ cycle. Qu'ont fait mes collègues philosophes ? Ils ont transformé la culture générale en cours de mathématiques et de logique, alors qu'une UV libre doit demeurer une UV libre ! Et la raison que j'entends alléguer à cette attitude - depuis quarante ans également - est que «la spécialisation doit commencer à la sortie du baccalauréat ». Seul le corporatisme de la profession soustend cette idée aberrante. Claude Allègre m'avait nommé au CNU : je n'y suis resté que quatre jours!

15 Je crois également que le champ social a son mot à dire sur l'orientation des disciplines. Il est regrettable que les philosophes continuent à gérer leur domaine comme s'ils étaient seuls au monde ${ }^{31}$. Ma concierge devrait être nommée au CNU ! Les universitaires se gèrent eux-mêmes au mépris de toute réalité sociale : plus un travail est académique, banal, répétitif, plus il reproduit la structure existante et plus il a de chances de permettre une carrière. Quoi de plus totalement anormal ? Je doute vraiment que l'université soit réformable de l'intérieur.

16 Il ne faut pourtant pas négliger les réformes entreprises, notamment la création par Lionel Jospin (lorsqu'il était ministre de l'Éducation nationale) de l'Institut Universitaire de France ${ }^{32}$, l'octroi des primes d'encadrement et de recherche doctorale, et les incitations au dynamisme de la recherche.

17 L. - L'UTLS, avec ses leçons magistrales, ne se présente-t-elle pas, d'une certaine façon, en « rivale » de l'Université ?

18 Y.M. - J'ai proposé dès le départ le principe des " leçons magistrales " car elles me semblent un bon moyen pour en finir avec le zapping ${ }^{33}$ intellectuel et les artifices des débats à la "Beaubourg" ${ }^{34}$ " qui dégénèrent vite en café du commerce. Les débats ne laissent en effet plus le temps aux intervenants de s'exprimer, ce qui lasse aussi bien les orateurs que leurs auditeurs. Beaucoup de conférenciers de l'UTLS sont heureux de pouvoir enfin disposer de près d'une heure pour leur intervention.

19 Mais, paradoxalement, l'UTLS ne fait pas concurrence à l'Université, car hormis en droit et en sciences économiques, les cours magistraux tendent à disparaître ${ }^{35}$. L'UTLS rappellerait donc plutôt l'université à ses missions ${ }^{36}$ : à la différence des séminaires de troisième cycle dont l'auditoire est averti et consensuel, l'UTLS s'adresse à un public plus hétérogène, étranger à tout discours prononcé sur le mode du « bien entendu » et de l'allusif.

20 L. - Mais si l'Université n'est pas réformable de l'intérieur, d'où peut provenir l'impulsion réformatrice ? Et quelles sont vos propres idées de réformes universitaires qui pourraient pallier la situation actuelle?

21 Y.M. - Si l'université ne se réforme pas d'elle-même, les changements proviendront directement du public : celui-ci s'adressera de plus en plus aux initiatives privées qui se multiplient sur le marché de l'offre d'enseignement. Ainsi, l'UTLS bénéficie d'ores et déjà d'une visibilité et d'un retentissement que le CNED (Centre National d'Enseignement à Distance) n'a toujours pas!

22 Je commence même à m'interroger sur l'autonomie de gestion des carrières universitaires et je suis moi-même étonné d'adopter une telle position! On a longtemps défendu une idée de l'université, d'origine allemande, fondée sur la capacité des professeurs à gérer eux-mêmes leur carrière et l'indépendance totale. Pas de recrutement politique, pas d'interférence du champ social et pas de pressions extérieures : telles étaient les 
conditions de l'indépendance universitaire. Or, après ce que j'ai observé tant au CNU qu'au CNRS, j'en viens à penser qu'il faut peut-être en finir avec l'indépendance universitaire telle qu'elle est maintenant pratiquée - tant le corporatisme est vivace et solide en France - et aussi impulser un large mouvement de décentralisation qui doit permettre une plus forte responsabilisation des acteurs.

La suppression des diplômes nationaux devrait être le corrélat de la nécessaire autonomie complète de l'université : il est temps, par exemple, de régionaliser l'agrégation. Il faut favoriser une ouverture au moins européenne au niveau des recrutements et des carrières. La mise en concurrence nationale et européenne causera probablement dans un premier temps de graves dysfonctionnements mais elle me parait indispensable. Cette ouverture existe déjà mais doit être renforcée et suppose une meilleure prise en compte des profils recherchés par les universités et de la diversité des formations proposées en Europe.

L'ouverture internationale vaut également pour les programmes. J'ai moi-même plusieurs fois été professeur à l'étranger ${ }^{37}$ et j'en retire plusieurs enseignements, notamment l'utilité d'importer des premiers cycles entièrement pluridisciplinaires, non pas à la carte, mais proposant plusieurs blocs de matières, comme c'est le cas en Allemagne, au Royaume-Uni et aux États-Unis. Pourquoi ne pas offrir à un étudiant français la possibilité de choisir à son entrée à l'université un DEUG composé d'un tiers de médecine, d'un tiers de philosophie et d'un tiers de sciences économiques ? Cette pluridisciplinarité serait un bon moyen d'entamer le corporatisme universitaire, dont je vous ai dit tout à l'heure que, selon moi, il était à l'origine de - et donc conforté par - la monodisciplinarité imposée dès l'entrée à la faculté. C'était un peu l'esprit d'une partie de la loi Bayrou ${ }^{38}$, mais elle n'a pas fonctionné et la réforme s'est transformée en usine à gaz.

Enfin, il faut supprimer les institutions type $\mathrm{CNRS}^{39}$ et remplacer les chercheurs à vie par des professeurs qui pourraient alterner les périodes d'enseignement et de recherche.

L. - Cette mise en concurrence est une remise en cause complète de notre modèle actuel, certes centralisé mais unitaire et égalitaire. Que répondez-vous à ceux qui vous accuseront d'accroitre les inégalités?

27 Y.M. - Je leur réponds que c'est d'ores et déjà le cas.

28 Dans les faits, les universités sont très inégales, et véhiculent des paradoxes qu'il est intéressant de souligner. Ce sont en effet souvent les universités modestes ou excentrées qui ont développé les formations les plus originales, notamment dans le domaine des sciences où l'on est moins prisonnier du centralisme parisien. Il existe ainsi des foyers d'excellence à Grenoble, Strasbourg, Nice, Toulouse ou Bordeaux qui ont développé des liens avec l'industrie et sont davantage lestés par le monde réel. Je ne dis pas que l'université doit systématiquement se tourner vers l'industrie, mais elle doit être guidée par la réalité. Or, tant qu'elle fonctionnera en circuit fermé, elle se privera de toute modernisation.

Et puis, il faut tout de même ne pas se leurrer : nous nous comportons tous de manière consumériste vis-à-vis de l'éducation. Tous les journaux publient régulièrement des classements des lycées ${ }^{40}$, des universités, des grandes écoles et en recensent les meilleurs. Les classes aisées peuvent faire leur marché car elles sont les mieux informées, mais les plus pauvres, elles, vont à l'université par défaut. 
30 L. - Ce consumérisme que vous décrivez suppose qu'il existe justement une offre de savoirs, à laquelle participe notamment l'UTLS. Considérez-vous que les savoirs sont suffisamment exploités, notamment à l'Université ? Et comment comptez-vous prolonger l'expérience de l'UTLS?

31 Y.M. - Il existe plusieurs initiatives, dont la vôtre, qui permettent une meilleure valorisation et exploitation des travaux réalisés par les étudiants. Je citerai également l'association récente du «Sélection Reader's Digest » avec les Éditions Larousse, qui publie certains mémoires de maîtrise d'excellente qualité ${ }^{\prime 1}$. Mais si l'important est de faire savoir qu'un certain nombre de choses existent, tout n'est pour autant pas bon à publier et il ne serait parfois pas inutile d'être plus scrupuleux sur la qualité ${ }^{42}$. J'ai ainsi le sentiment qu'en matière de recherche, beaucoup de progrès ont été réalisés (de mon temps il n'y avait pas d'allocations de recherche ni de bourses doctorales) mais que dans le même temps l'information et la valorisation des travaux de recherche demeurent insuffisantes.

Vers le 15 juin 2000, les 40 premières conférences de l'Université de Tous les Savoirs verront le jour sous la forme d'un ouvrage publié aux éditions Odile Jacob. Bien que le compte rendu de celles-ci soit déjà disponible sur Internet, nous faisons face à une véritable demande spontanée, à laquelle j'estime nécessaire de répondre. Le public se comporte de plus en plus de manière consumériste dans nos sociétés : on peut trouver cela bien ou mal, mais c'est une réalité. À travers le consumérisme se traduit une demande sociale qui mérite d'être prise en compte ${ }^{43}$.

33 Un réel engouement s'est créé autour de l'UTLS : elle a déjà rassemblé plus de 50000 personnes les deux premiers mois de l'année. Je ne connais pas bien ce public qui est venu au CNAM et, ce qui m'intéresse, ce sont aussi bien les gens qui consultent Internet ${ }^{44}$ que ceux qui écoutent des interviews des conférenciers de l'UTLS à la radio ${ }^{45}$. En fait, nos publics sont très différents et très segmentés ${ }^{46}$, ce qui me fait dire que l'UTLS est comme un produit à déclinaisons multiples. Ainsi, certaines personnes ne se rendent au CNAM ou sur le site internet qu'une seule fois, sur un sujet précis qui les concerne ; d'autres sont intéressées par le large éventail de thèmes proposés. Nous avons également répondu à de très nombreux appels provenant d'organismes à l'étranger (alliances françaises, centres linguistiques français à l'étranger) qui nous demandent de leur transmettre les textes et les vidéos de nos conférences. L'UTLS révèle un consumérisme à deux faces : une commercialisation de la société mais en même temps une forme de la démocratie. Ce que j'en retiens, c'est qu'on ne vend pas aux gens ce dont ils ne veulent pas.

34 Nous comptons aussi prolonger l'expérience de l'UTLS, probablement pas sous une forme quotidienne - très lourde à organiser - mais sans doute en ligne, sur Internet, qui est d'ailleurs appelé à se développer considérablement dans le champ de l'offre éducative. Connaissez-vous http://www.fathom.com ? Il s'agit d'un nouveau site qui rassemble notamment la Columbia University et la London School of Economics (LSE) ${ }^{48}$ et va commercialiser différents produits d'enseignement.

L. - N'y a-t-il pas un paradoxe entre votre discours ici réaliste, cynique, voire révolutionnaire, et l'« emballage " de votre projet, un peu " ringard ", au sens où il évoque la vulgarisation scientifique telle qu'elle s'est fortement développée au XIX ${ }^{\mathrm{e}}$ siècle et sous la III ${ }^{\mathrm{e}}$ République, et qui n'est pas sans renvoyer à une conception de l'éducation périmée et étrangère à la vôtre ? Si l'UTLS suscite une telle demande de la part du public, c'est peut-être avant tout une demande de sens que celui-ci formule. Peut-être est-il attiré 
par l'aspect idéaliste et utopique de la vulgarisation « vieille école » de l'UTLS ? Ou bien par le côté dramatique de ce qui a toutes les allures d'un parcours initiatique, d'une Odyssée intellectuelle pour « Ulysses » contemporains? Mais vous ne pouvez pas combler ces aspirations car bien entendu, vous n'êtes pas là pour donner un sens à l'existence des gens. N'avez-vous pas le sentiment de tromper votre public?

Y.M. - Il est vrai que l'UTLS se développe sous les dehors de la vulgarisation du XIX ${ }^{\mathrm{e}}$ siècle, mais, pour être honnête, au début, nous-mêmes ne savions pas où nous allions, et c'est pourquoi nous nous sommes d'abord raccrochés à ces vieux repères, à ces cadres traditionnels : c'est seulement ensuite et au fur et à mesure que nous avons investi le projet de nos ambitions propres, et donc passablement différentes de celles correspondant aux " habits » du hussard noir vulgarisateur du XIX siècle. Donc, à l'heure qu'il est, effectivement, je " trompe » les gens, mais en tablant aussi sur le fait qu'ils ne sont pas dupes et qu'ils se rendent bien compte que c'est toujours dans les vieilles outres que l'on fait le meilleur vin.

Cette méthode, que j'ai apprise lorsque je dirigeais l'École des Beaux-Arts, est issue du constat que l'on ne peut rien faire en France en proclamant immédiatement que c'est nouveau ${ }^{49}$. Il faut toujours simuler d'agir dans la continuité. Quand je suis arrivé aux Beaux-Arts, j'ai commencé par dire et redire « il n'y aura pas de réforme ». C'est au fur et à mesure que vous faites réellement les réformes (parallèlement à un discours qui les nie) que vous devez réfléchir et les infléchir en fonction de vos réflexions.

Cette analyse vient de Nietzsche. J'ai toujours été inspiré par cette idée de la Généalogie de la Morale selon laquelle une force ne peut vaincre qu'en prenant le masque d'une autre force $^{50}$. L'une des rares choses que j'emprunterais à Deleuze ${ }^{51}$ est cette idée nietzschéenne selon laquelle le nouveau doit se profiler sous l'aspect de l'ancien pour avoir le temps de s'implanter. Surtout dans un pays comme la France qui déteste le changement, à la différence des ÉtatsUnis ${ }^{52}$. Claude Allègre avait beaucoup de bonnes idées mais il aurait dû ne rien dire ! L. - Les nouvelles technologies amplifient considérablement la diffusion des savoirs. Faut-il pour autant en savoir toujours plus ? Et sur quoi débouche cette course folle des savoirs dont participe aussi l'UTLS (et qu'illustre remarquablement Nicolas Vial ${ }^{53}$ )?

Y.M. - Selon moi, cette situation nouvelle présente trois caractéristiques majeures. D'une part, les nouvelles technologies mettent à la disposition du public un véritable tohu-bohu d'offres exceptionnelles : tout et n'importe quoi est désormais accessible. D'autre part, l'absence de critères de vérification avantage les offres les plus dynamiques mais pas forcément les plus sérieuses. Enfin, l'absence d'instance de conseil, autrement dit de prescripteur, nous installe dans une situation inédite. Résumons donc : l'offre est énorme mais elle s'accompagne d'un déficit de vérification et d'un réel manque de prescripteurs.

Internet présente encore d'importants dysfonctionnements. Prenons par exemple les sites de philosophie : beaucoup de professeurs prennent l'initiative de construire des sites très dynamiques mais sans grand intérêt ! A contrario, je peux citer l'exemple d'un ingénieur de l'École supérieure d'électricité qui développe un site de philo : il est visiblement autodidacte, mais son site est intéressant et propose de très nombreuses conférences et séminaires en ligne, ainsi qu'une ébauche de répertoire des philosophes français. Aussi faut-il faire confiance à un certain "darwinisme ", en ce domaine, qui devrait permettre d'établir une "sélection naturelle " en fonction des besoins des utilisateurs. 
L. - Pensez-vous que les intellectuels servent encore à quelque chose?

Y.M. - Pour le moment, les intellectuels sont dans une mauvaise posture. D'en haut, le prescripteur ne peut rien dire : il faudrait déjà qu'il ait connaissance de tout ce qui existe ! Mais on constate en fait que se reconstituent spontanément des réseaux de prescription indispensables pour s'y retrouver ${ }^{54}$, comme la technique de labélisation ${ }^{55}$, que pratiquent la LSE ou la Columbia University lorsqu'ils « cautionnent » un site : c'est finalement à qui obtiendra les «credentials » les plus importants et les plus pertinents.

L. - Pour conclure, ne pensez-vous pas que le savoir doit aussi renvoyer à la notion de responsabilité et qu'en éduquant il devient le meilleur garde-fou contre l'ignorance, les préjugés et l'arbitraire qui caractérisent parfois nos sociétés ? D’une certaine façon, le savoir et la démocratie ne sont-ils pas interdépendants?

Y.M. - Une idée très traditionnelle loue l'éclairement, qui permet à tout un chacun de s'orienter $^{56}$, d'avoir des doutes et de sortir du confinement des idées. Au-delà d'une position passive d'admiration et de réception, le savoir doit nous permettre de réfléchir.

6 Si je prends l'exemple d'une personne qui a bien suivi l'ensemble des conférences de l'UTLS sur la génétique, je pense qu'elle aura non seulement une idée des risques encourus par les manipulations génétiques mais aussi quelques doutes sur la sélection et l'eugénisme. Le savoir est donc libérateur, mais le questionnement sur le savoir l'est aussi. ${ }^{57}$. C'est un effet à double détente : le savoir n'est pas tant ce qui vient délivrer les gens ${ }^{58}$, mais plutôt un outil préalable à la comparaison et à la réflexion.

4 La démocratisation de nos sociétés se caractérise par le fait que ces dernières admettent des niveaux de réflexion extrêmement différents et de qualité très variable ${ }^{59}$. D'une certaine manière, il n'est pas souhaitable - ni même, comme on l'a vu, possible, dans le cadre des technologies nouvelles - que les intellectuels ${ }^{60}$ " fassent le tri » des savoirs pour nous, sans quoi ils reprendraient une position d'autorité. Dans le même ordre d'idées, Claude Allègre disait que si la science fonctionnait démocratiquement, elle ne changerait jamais.

Je pense qu'il n'y a pas de bonne manière de guider la réflexion, et j'aime assez cette image - peut-être vieillotte ! - du Larousse qui « sème à tout vent ${ }^{61}$ ". L'UTLS participe en quelque sorte de la même idée : une ballade féconde en étonnements et en surprises. Des orateurs s'expriment sans que l'on sache ce que cela donnera. L'UTLS va certainement conforter certaines personnes âgées dans leur dogmatisme ou dans leurs angoisses ; d'autres auditeurs ne comprendront rien à la conférence mais en sortiront motivés pour le restant de leurs jours ! Ce hasard des réceptions est fondamental, car c'est peut-être entre les savoirs que finalement les choses se passent.

\section{ANNEXES}

Entretien réalisé par François-Xavier Priollaud, titulaire d'un DEA de droit public, et Audrey Techer, doctorante en philosophie à Paris I. 


\section{NOTES}

1.Une conférence par jour, chaque jour de l'année 2000, au Conservatoire National des Arts et Métiers de Paris (292, rue Saint-Martin, 75003 Paris, Amphithéâtre Paul Painlevé), à $18 \mathrm{~h} 30$ les jours de la semaine, et $11 \mathrm{~h}$, les samedi, dimanche et jours fériés. Sur une idée originale de Jean-Jacques Aillagon, Président du Centre Georges Pompidou et de la Mission 2000 en France, conçue et mise en œuvre par Yves Michaud, Professeur de Philosophie à Paris I, l'Université de Tous les Savoirs est le versant réflexif, la contrepartie intellectuelle, des festivités de l'an 2000 organisées par la Mission, comme « rites » de passage à l'an 2000 : spectacle des Roues, Portes de l'an 2000 (lors de la nuit du 31 décembre 1999), mais aussi la Méridienne Verte, Fraternité 2000, etc.

2.Le choix du CNAM, voulu par Y. Michaud, renoue en effet symboliquement avec le projet de l'Encyclopédie, qui était une Encyclopédie des Sciences, des Arts et des Métiers. Le CNAM répond à l'origine à l'idée de créer un « Conservatoire » présentant le spectacle de machines et instruments techniques, une sorte de ménagerie pour machines et de zoo pour l'intelligence humaine, d'où devaient procéder divertissement, promotion des inventeurs, et instruction. En 1794, sur le rapport de l'abbé Grégoire (1750-1831), la Convention décida de réunir tous les objets propres à la démonstration des arts industriels dans un « dépôt de machines, modèles, outils, dessins, descriptions et livres dans tous les genres d'arts et métiers ", afin qu'on y expliquât « la construction et l'emploi des outils et machines utiles aux arts et aux métiers ", et qu'on y recueillît « l'original des instruments et machines inventés ou perfectionnés ", dépôt installé dans les bâtiments de l'ancien prieuré de Saint-Martin-des-Champs, dont il reste l'église et le réfectoire. C'est seulement par l'ordonnance du 15 décembre 1819 que le CNAM devint « une haute école d'application des connaissances scientifiques au commerce et à l'industrie » délivrant un enseignement " public et gratuit pour l'application des sciences aux arts industriels ». Actuellement considéré comme l'un des organes les plus efficaces de l'information et du jugement technologiques de l'expansion industrielle en France, le CNAM, « grand établissement d'enseignement supérieur et de recherche », sous la tutelle du Ministère du même nom, cumule de manière originale trois statuts : 1) un centre de formation de l'industrie et du tertiaire 2) un Institut de recherche pluridisciplinaire 3) un Conservatoire au Musée et à la Bibliothèque en sciences, arts et techniques prestigieux (cf. aussi le site web du CNAM : http://www.cnam.fr).

3.Alors que, dans nos sociétés contemporaines, le savoir est désormais éclaté, intotalisable, se développant dans plusieurs directions, ce qu'Y. Michaud tient pour acquis.

4.Il n'y a par exemple rien sur la psychanalyse (les auditeurs de l'UTLS le reprochent bien des fois), et ce de manière délibérée : Y. Michaud s'en explique en disant que, pour sa part, il souhaitait une leçon sur les thérapies mentales en général, mais il y a malheureusement trop d'écoles et de conflits de méthodes et de « chapelles » en ce domaine pour pouvoir trouver un spécialiste suffisamment ouvert pour traiter un sujet si général... Mais la contrepartie de ces options fermes et tranchées est la programmation du mois de décembre qui se fera en fonction des suggestions du public.

5.Par exemple, l'apparition de deux nouvelles disciplines : la biochimie, et la connaissance du globe, qui ont contribué à redéfinir de manière radicale l'espace du savoir. 
6.Le projet de l'UTLS se situe donc à mi-chemin entre la superficialité médiatique - par son désir d'échapper à l'obsolescence rapide, aux sujets à la mode, au quotidien des événements et à l'inessentiel, qui caractérisent par trop toutes les velléités de pensée au présent - et la " pensée pour l'éternité », ou prétention de profondeur : écueil sur lequel échouent en général tous les philosophes. Y. Michaud, qui fait souvent lui-même œuvre de philosophie au présent dans ses ouvrages, prend toujours soin d'élaborer des méthodes et des garde-fous à la première tentation; quant à la prétention à penser « de tout temps » : « laissons cela aux autres », dit-il un jour, lors d'un séminaire de philosophie, stigmatisant ainsi certains de ses collègues...

7.Le mystère de sa conception peut être résumé ainsi: par une stratégie en " entonnoir » (comme dirait le philosophe Gilbert Simondon), Y. Michaud a consulté très largement la communauté savante francophone à travers les grands organismes de recherche (Académies, Universités, Instituts, mais aussi entreprises, etc.) en demandant à chacun quels étaient les sujets indispensables à traiter dans leur domaine : 1700 questions obtenues, que des comités restreints de tri ont alors travaillé à réduire à 335 sujets (puisque le programme de l'UTLS ne court que jusqu'au 30 novembre : le mois de décembre sera programmé en fonction des suggestions du public) en respectant à peu près l'équilibre entre les disciplines. Cette manière de faire s'oppose à la 1re idée qu'il avait eue : confier d'emblée la programmation à des comités, discipline par discipline ; l'obstacle rencontré alors : les gens qui ont de la compétence n'ont pas forcément du temps pour les comités, et les gens qui ont du temps pour les comités n'ont plus forcément de la compétence.

8.Elles s'inscrivent d'ailleurs dans un module au titre bien impertinent, qui a même choqué plus d'un conférencier de sciences humaines invité à y parler : « Deuxième étape de réflexion et de critique : y a-t-il encore des sciences humaines?».

9.Parce qu'avec les habitudes de réflexion intellectuelle française, déclare par ailleurs $Y$. Michaud, on se serait vite retrouvé avec le contraire : $2 / 3$ de sujets de sciences humaines, et $1 / 3$ de sujets scientifiques... Il y a donc, au départ, il est vrai, une option clairement " positiviste ", mais pas pour autant « scientiste », à l'origine du programme de l'UTLS, car les conférences scientifiques problématisent - pour la plupart - les sciences. Par ailleurs, Y. Michaud, malgré sa formation et ses centres d'intérêts philosophiques, est persuadé que les progrès des sciences et les changements relatifs aux conditions matérielles de la vie qu'ils induisent, pèsent probablement plus lourd, en termes d'évolution sociale et politique, que la « pure » idéologie...

10.Dans le cadre du module "Justice, responsabilité et contrat : le droit en mouvement » (20-22 février), on a pu entendre successivement Antoine Garapon sur « les fonctions rituelles de la justice », Geneviève Viney sur « la responsabilité et ses transformations », et Alain Supiot sur « la contractualisation de la société ».

11.Par exemple, Anne Fagot-Largeault sur « L'éthique de l'investigation scientifique sur l'être humain » (20 janvier), ou Marion Guillou sur « La sécurité des aliments : prévention, surveillance, crises » ( $1^{\mathrm{er}}$ mars), etc.

12.Vous pouvez retrouver les découpages disciplinaires du CNRS sur son site web : http:// www.cnrs.fr et ceux du CNU sur le site web suivant : http://garp.univ-bpclermont.fr/guilde, qui n'est autre - d'ailleurs - que le site de « La Guilde des Doctorants », proposant en ligne les informations et les conseils les plus divers et précis à l'usage de tout étudiant en thèse, ce qui ne saurait manquer d'intéresser les lecteurs de la revue Labyrinthe...

13.Depuis l'Antiquité (Pline l'Ancien) jusqu'à Auguste Comte, en passant par Descartes, Bacon, Leibniz, Diderot et d'Alembert, etc., la mise en ordre du savoir, en vue de l'exposer et 
de l'enseigner est une obsession des philosophes, sinon du genre humain tout entier. Des découpages en objets, disciplines, rubriques, académies, qui nous paraissent aujourd'hui évidents, et sur lesquels nous ne nous interrogeons même plus, ont une histoire, un lien direct avec notre culture occidentale qui s'est toujours efforcée d'organiser logiquement le savoir (cf., par exemple, le mode de classement des bibliothèques ou même des archives de grands journaux, ou bien encore de sociétés de documentation), et un rapport avec toutes les problématiques relatives au langage (quel est le langage suffisamment idéal pour permettre d'apprendre logiquement et rapidement le plus de choses possibles? cf. les réflexions de Descartes à ce sujet et de Leibniz, par exemple). L'Ordre du savoir a déjà été démonté dans son histoire et les relations du pouvoir qui le créent par Michel Foucault, dans Les Mots et les Choses (Gallimard, 1966). À l'égard donc de cette tradition, il est intéressant d'observer que le découpage d'Y. Michaud, qui, certes, ne se prend pas trop au sérieux, suit une voie originale (dynamique, loin des acquis lourdement institutionnalisés) : de la vie dans ses constituants chimiques les plus infimes, jusqu'à l'homme, puis tous les phénomènes qui le dépassent (grand vertige des sciences dures prévu pour cet été), pour finalement revenir à l'homme et à ses problèmes de vie en société, et se clore par une partie « interactive » à laquelle personne n'avait jamais pensé auparavant : l'Encyclopédie du XXI ${ }^{e}$ siècle se termine par l'université de tout ce que les gens veulent savoir...

14.Aussi Y. Michaud va-t-il chercher hors de France des sociologues qui conceptualisent (Anthony Giddens), et qui sont presque des « philosophes » dans leur approches (comme Zygmunt Bauman, sociologue britannique d'origine polonaise, et d'inspiration lévinassienne, qui intervient le 7 mai à l'UTLS pour parler de "l'identité »; il est l'auteur de Postmodern Ethics, Blackwell, 1993, Life in Fragments, Blackwell, 1995, et Le Coût humain de la mondialisation, Hachette Littératures, 1999, seul ouvrage traduit en Français). 15.Y. Michaud a ainsi sollicité Françoise Parot pour prononcer une conférence au titre éloquent : « La psychologie : les conditions de la survie » (1 $1^{\mathrm{er}}$ avril). Françoise Parot est maitre de conférences d'Histoire de la psychologie à l'Université René Descartes (Paris V) et chercheur associée de l'équipe de Recherches Épistémologiques et Historiques sur les Sciences Exactes et les Institutions Scientifiques (REHSEIS), Paris VII/CNRS. Elle a travaillé sur l'histoire de la représentation contemporaine de l'esprit, sur l'étude de la construction des problématiques contemporaines de la psychologie, et s'intéresse actuellement à l'activité onirique, à la croyance dans les pouvoirs de l'esprit sur la matière ou à lui survivre, et sur les représentations que les sujets se font de leur identité. Directrice de la collection « $1^{\mathrm{er}}$ cycle de Psychologie » aux PUF, elle a publié : Dictionnaire de Psychologie (PUF, 1990, réédition 1999), L'Homme qui rêve (PUF, 1995), Introduction à la psychologie. Histoire et Méthodes (PUF, 1998), et Existe-t-il une nature humaine? Psychologie historique, objective, comparative (Synthélabo, coll. « Les Empêcheurs de penser en rond », 2000).

16.Y. Michaud est critique d'art depuis 1975, et a rédigé nombre de préfaces de catalogues d'artistes et d'articles dans des revues d'art contemporain parmi les plus prestigieuses : Les Marges de la Vision. Textes Critiques 1978-1995 (Jacqueline Chambon, coll. « Critique d'art ", 1996) regroupent de nombreuses critiques, qui permettent de se faire une idée de son expérience dans le domaine de l'art, - expérience d'ailleurs également forgée au poste de rédacteur en chef des Cahiers du Musée National d'Art Moderne (Centre Georges Pompidou), qu'il a occupé de 1986 à 1990.

17.Il est le premier à la mettre en œuvre dans ses travaux : l'histoire, l'histoire de l'art, la sociologie, l'économie, la philosophie, etc. sont ainsi régulièrement convoquées dans ses 
ouvrages, comme par exemple dans Violence et Politique (Gallimard, coll. « Les Essais », 1978) ou plus récemment dans La Crise de l'art contemporain : utopie, démocratie et comédie (PUF, coll. « Intervention philosophique », 1997). Dans ce dernier ouvrage, par exemple, Y. Michaud pratique la pluridisciplinarité en vue d'un seul but : élucider le mystère de la « crise de l'art contemporain » actuelle. Ainsi, pour expliquer une situation apparemment insoluble où partisans et adversaires férus de l'art contemporain s'empoignent violemment et stérilement, il convoque la réalité des faits (donnée par l'histoire, la sociologie), analyse les positions des uns et des autres, utilise les concepts de philosophes (Kant, Habermas) et parvient à donner une interprétation politique de cette crise, en ayant ainsi tenu ensemble, tout au long, «faits et représentations ». Cette crise de l'art contemporain est en fait une crise de notre représentation de l'art, laquelle n'est autre que

démocratique, car liée depuis les débuts de la $\mathrm{III}^{\mathrm{e}}$ République, aux idéaux devenus illusoires de celle-ci (promotion culturelle de tous, égalité énonçant que chacun doit pouvoir donner son avis sur l'art, etc.). L'art n'a peut-être pas, peut-être plus, à être démocratique de nos jours, à plaire à tout le monde, à être accessible à tous ; d'autres moyens de cohésion politique existant et étant plus efficaces pour remplir ce rôle.

18.La pensée ne sort pas toute casquée du cerveau, telle Athéna de la tête de Zeus... Contre la représentation de la pensée comme étant « inspirée », trop souvent véhiculée par les philosophes qui dissimulent les « coulisses » de leur propre réflexion, Yves Michaud, un peu dans la tradition cartésienne, admet honnêtement qu'il y a des apprêts, des techniques, du « bricolage » dans la pensée, et pour penser. Philippe Dagen - le critique d'art du Monde - qui l'a « combattu » âprement sur la question de l'art contemporain, ne l'a-t-il pas d'ailleurs appelé, comme dans une étincelle de lucidité, « le philosophe-ingénieur » (cf. Le Débat, n 98, janv-fév. 1998, Philippe Dagen : « Sur Jean Clair et Yves Michaud », p. 13-19, citation p. 16)?

19.Sous cette formulation («boîte à outils»), l'idée qu'énonce Y. Michaud a en fait été élaborée par Michel Foucault. Évoquant cette idée, Foucault se justifiait de ne pas faire de "l'histoire de la philosophie », pour elle-même, en suivant pas à pas la pensée d'un auteur, et revendiquait au contraire de puiser dans les auteurs - un peu arbitrairement au besoin - ce qu'il voulait pour " construire autre chose », une réflexion personnelle : celle-là même étant susceptible à son tour, de se transformer en « boîte à outils " pour qui la reprendra et l'investira d'un nouveau but (cf. Dits et Écrits 1954-1988, 1994, Gallimard, coll. «Bibliothèque des Sciences Humaines », tome 2, texte 151, p. 720). Mais ce que veut dire en réalité Y. Michaud en se référant à Wittgenstein, c'est que les différentes manières de penser sont comme des « jeux de langage » (notion wittgensteinienne, cf. Investigations philosophiques, Gallimard, coll. « Tel », 1986), c'est-à-dire des « outils » langagiers inventés pour décrire ou se situer dans une sphère spécifique (par exemple, le monde de l'art, et, à l'intérieur du monde de l'art, le cercle des admirateurs férus de Picabia) et que l'on peut utiliser ailleurs, exporter dans d'autres sphères, où ils sont susceptibles de faire voir - en les nommant - des aspects jusque-là inpensés. C'est la réflexion qu'Y. Michaud développe dans son ouvrage : Critères esthétiques et jugement de goût (Jacqueline Chambon, coll. « Rayon Art », 1999), dans lequel il traite justement comme « outil » le concept philosophique de « jeu de langage » lui-même, afin de décrire la situation actuelle de l'art contemporain, sur le plan de sa réception : des façons dont il est appréhendé socialement, et de celles dont on peut l'appréhender (cf. p. 70-102).

20.Lors de la conférence qu'il a donnée pour l'UTLS sur « L'histoire » (4 avril). 
21.On peut d'ailleurs caractériser la manière philosophique d'Y. Michaud par une attention scrupuleuse au réel, alimentée précisément par cette constante pratique de la pluridisciplinarité qui lui fait recueillir des données concrètes issues aussi bien de la sociologie, de l'histoire ou de l'économie, réel dont il tire ensuite une conceptualisation philosophique fine et pertinente, d'autant plus qu'elle a été dérivée du réel et non " plaquée » sur lui, comme tant de philosophes ont l'habitude de le faire. Pour ne donner qu'un seul exemple, il a inventé le concept de « nervosité » pour rendre compte d'un des traits de l'individu contemporain, (de l'identité contemporaine) dont tout l'environnement (perte de la référence à l'espace en liaison avec nouvelles technologies et médias, perte de la référence au temps et vécu au présent continuellement, perte des normes de conduites et de toute autorité pouvant les édicter et les cautionner, caractérisant notre société et les individus qui la composent) fait qu'il « oscille sans cesse entre la jouissance excitée et la panique » et qu'il est sans cesse dans une situation où il « se débrouille ", sans jamais être fixé quelque part, ni assuré de ce qu'il fait, encore moins sûr de ce qu'il est (cf. La Violence Apprivoisée, Hachette, coll. «Questions de Société », 1996, p. 88-91, qui contient les observations concrètes à l'origine de sa réflexion).

22.Pour pallier cette déficience, Y. Michaud s'est associé à Jacqueline Chambon, et dirige dans sa maison d'édition, depuis 1989, la collection « Rayon Art » qui s'est aujourd'hui imposée comme l'une des plus dynamiques dans le domaine de l'édition d'essais sur l'art. Dans un entretien au Monde (30 janvier 1996), il déclarait que cette collection se caractérisait par la «notion d'éclectisme polémique : multiplication des disciplines, histoire sociale de l'art, psychanalyse, analyse formelle, analyse philosophique », voilà pour l'éclectisme ; et " polémique, c'est-à-dire ayant une sorte de pertinence agaçante ». Sont parus, pour ne citer que quelques ouvrages : Hans Belting, L'Histoire de l'art est-elle finie? (1989), Michael Baxandall, Les Formes de l'Intention (1991), Nelson Goodman, Les Langages de l'art (1990) et Manières de faire des mondes (1992), Harold Rosenberg, La Dé-définition de l'art (1992), Ernst Gombrich, Réflexions sur l'histoire de l'art (1992), etc.

23.Présidée par Jacques Revel, l'EHESS est un " grand établissement » (depuis 1984) d'enseignement supérieur, relevant du Ministère de l'Éducation Nationale, de la Recherche et de la Technologie. Elle est issue de la VIe section de l'École Pratique des Hautes Études, à laquelle elle a succédé en 1975. Elle a pour mission la recherche et l'enseignement de la recherche en sciences sociales, et l'étude des rapports des sciences sociales avec les sciences de la nature et de la vie. Les cours dispensés couvrent les principaux domaines des sciences sociales : histoire, économie, sociologie, anthropologie sociale, ethnologie, démographie, géographie, archéologie, psychologie, linguistique, droit et société, philosophie et épistémologie, mathématiques sociales, méthodes statistiques, informatiques, et graphiques pour les sciences sociales. Elle dispose de plusieurs centres en France (cf. http://www.ehess.fr).

24.Sciences Po est né en 1872 lorsque Émile Boutmy a créé l'École Libre des sciences politiques pourrenouveler la formation des élites en France. Le but de l'École était de dispenser une formation pluridisciplinaire de haut niveau centrée sur l'analyse du monde contemporain. En 1945, l'État reprit l'œuvre de Boutmy en créant d'une part la Fondation Nationale des Sciences Politiques (FNSP, président actuel : René Rémond) et l'Institut d'Études Politiques de Paris (IEP, président actuel : Richard Descoings), pour « favoriser le progrès et la diffusion des sciences politiques, économiques et sociales », et lui conféra le statut de " grand établissement » en 1984 (cf. http://www.sciences-po.fr).

25.Un peu moins connu sans doute, le Collège International de Philosophie (président : Jean-Claude Milner) est une institution autonome de recherche et de formation à la 
recherche, ouverte à tous, sans condition de titres ou de diplômes, fondée le 10 octobre 1983 à Paris, fonctionnant sous le régime d'association privée, et qui répond à un projet conçu par des philosophes, après une large consultation de la communauté intellectuelle française et internationale. Hébergé dans les locaux du Ministère de l'Éducation, de la Recherche et de l'Enseignement Supérieur (1, rue Descartes, 75005), il propose des séminaires (et aussi conférences, débats, forums, colloques, etc.) en libre accès (un peu comme au Collège de France), où la philosophie est sans cesse associée à, croisée avec - de manière libre et « expérimentale » pour ainsi dire - d'autres disciplines, par des chercheurs de tous horizons philosophiques, disciplinaires, nationaux, etc. : art, littérature, droit, économie, psychanalyse, sciences sociales, sciences, mais aussi « intersciences ", c'est-à-dire ces activités théoriques qui n'ont pas encore de place délimitée dans la cartographie des savoirs et l'espace des institutions académiques. Il délivre aussi éventuellement un diplôme, et beaucoup de professeurs de philosophie qui y exercent se voient décharger de leurs horaires en lycée et universités. On peut en trouver le programme sur le site de l'Université de Paris VII : http ://www. diderotp7.jussieu. fr/philo), qui prête également ses locaux.

26.L'on peut témoigner en effet de la marginalité d'Y. Michaud professeur de Philosophie, et qui est en partie due à sa pratique de la pluridisciplinarité (ou de l'originalité ?) dans ses séminaires à Paris I : ainsi, il est le seul à consacrer des cours de philosophie politique à des sociologues, étrangers, et inconnus en France, même de ses pairs !

27.Dans le même entretien au Monde (30/01/96), Y. Michaud dénonçait déjà « le corporatisme des universitaires » et « une connivence généralisée devant la peur des réalités ".

28.Allusion au célèbre ouvrage de Pierre Bourdieu et Jean-Claude Passeron (d'ailleurs intervenant de l'UTLS, le 2 avril, sur « le raisonnement sociologique »), La Reproduction. Éléments d'une théorie du système d'enseignement (Minuit, coll. « Le sens commun », 1970).

29.À la crise universitaire de mai 1968 répondit la loi Edgar Faure, votée à la quasiunanimité le 12 novembre 1968. Celle-ci a remodelé le système universitaire français sur les principes d'autonomie, d'interdisciplinarité et de participation. Les anciennes facultés, mono-disciplinaires, firent place à des Unités d'Enseignement et de Recherche (UER) (cf. Titre II) regroupées au sein d'universités, nouvellement créées, indépendantes (Titre III, IV et V) et pluridisciplinaires (la vieille Sorbonne est découpée et de nouveaux établissements ouvrent leurs portes : Vincennes, Dauphine, Villetaneuse, etc.). Les étudiants sont appelés à la cogestion (Titre III, et IV), la politique reçoit droit de cité dans l'enceinte universitaire... Ce fut un changement radical du système universitaire français (Cf. Michel Winock, « Mai 68 ", dans La Fièvre hexagonale. Les grandes crises politiques 1871-1968, Seuil, coll. « Points Histoire », 1987, p. 327-373); et texte de la Loi n $68-978$ du 12 novembre 1968, dite « Loi d'orientation de l'enseignement supérieur », consultable in extenso sur : http://garp.univ-bpclermont. fr/guilde/Textes/Txtfond/L68-978.html; cf. aussi $h t t p: / / w w w . s o r b o n n e . f r)$.

30.En gestation entre octobre 1995 (mouvement étudiant de l'automne 1995) et février 1997 (tenue d'« États généraux » de l'université), la réforme du ministre de l'Éducation d'alors, François Bayrou, avait pour but une re-conception de l'organisation des premiers cycles universitaires, centrée autour du refus de la sélection à l'entrée de l'université et de la « logique de l'échec » des étudiants de 1er cycle, et projetait plusieurs mesures allant dans ce sens : pluridisciplinarité des enseignements, modules professionnels, stages d'apprentissage dans le monde du travail, tutorat (elle prévoyait aussi une amélioration de la « mobilité » entre universités et grands organismes, des « allocations sociales 
d'études ", ainsi qu'une délocalisation territoriale et une ouverture aux universités étrangères).

31.C'était l'opinion du militant communiste Paul Nizan, qui écrivit le célèbre Chiens de Garde (texte de 1932 ; réédité en 1965 chez Maspéro), dans lequel, au début de ce siècle, il stigmatisait les penseurs éthérés - y compris les plus brillants de la tradition philosophique : Bergson, Brunschwig, etc. - parce qu'ils philosophaient au mépris de la réalité sociale, sans faire en sorte que leur pensée trouve une accroche dans le réel (fondement ou effet), sans qu'elle puisse servir, dans la vie de tous les jours, à n'importe quel ouvrier. Au-delà de l'enracinement communiste, on peut noter la pertinence de cette remarque (que même des gens de droite pourraient formuler !), qui revêt plus de profondeur qu'elle n'en a l'air. On a en effet vu comment, loin de se détacher du réel, de la réalité sociale pour philosopher, Y. Michaud l'étudiait scrupuleusement (en déployant d'ailleurs des outils pluridisciplinaires) avant de la conceptualiser. Plus la peine d'être marxiste de nos jours pour pratiquer la philosophie ainsi : c'est juste une question de bon sens, dirait Y. Michaud.

32.L'Institut Universitaire de France - qui n'a pas de réalité physique, mais est juste un statut dérogatoire - est un système de reconnaissance des mérites particuliers de certains enseignants leur attribuant : $3 / 5$ de décharge de service, des crédits annuels à des fins de recherche (pour 5 ans), et dont le quota favorise explicitement les provinciaux. Outre une aide à la recherche, cet Institut constitue également un enrichissement en enseignement, puisque, après un an, un poste est créé à l'Université pour remplacer l'enseignant dont les horaires ont ainsi été allégés. En 1998 et en 1999, Y. Michaud a d'ailleurs été membre du jury senior de l'Institut Universitaire de France.

33.Y. Michaud développe par ailleurs cette idée selon laquelle « un nouveau régime de perception et d'expérience » est né dans nos sociétés contemporaines, " où prédominent le typique, les effets de reconnaissance, l'inattention, le balayage rapide d'une information réduite ", " proche du clip, du zapping, du voyage supersonique, $d u$ déphasage horaire et du tourisme ", qu'on peut difficilement ignorer, et ne pas mettre en relation, plus particulièrement, avec « la fin de la peinture et de la sculpture », puisqu'il traite de ces phénomènes dans un essai sur l'art, et où l'on voit - au passage - comment les considérations psychologiques, sociologiques et sociales viennent préciser et conforter un point de vue sur l'art (L'Artiste et les Commissaires. Quatre essais non pas sur l'art contemporain mais sur ceux qui s'en occupent (Jacqueline Chambon, coll. « Rayon Art », 1989, cf. p. 26).

34.Par ce nom générique, Y. Michaud veut désigner la mode actuelle des débats organisés à la télévision, à la radio, mais aussi bien dans ces lieux de transmission de savoir que se veulent la Villette, le Centre Georges Pompidou ou la Bibliothèque de France. Selon lui, pour faire un bon débat, il ne faut pas plus de trois intervenants et un bon animateur, qui laisse chacun s'exprimer en son temps, et pertinemment. C'est le principe même de l'art de la conversation, que plus personne ne pratique aujourd'hui, et qui est une idée des Lumières, née en opposition au long discours de l'homme d'Église. Le débat contemporain, qui ne laisse s'exprimer personne, où les intervenants ne s'écoutent même pas les uns les autres et parlent souvent de choses différentes, est la négation absolue de l'art de la conversation (cf. sur l'art de la conversation, les réflexions de Quentin Skinner ou de Marc Fumaroli, d'ailleurs intervenant de l'UTLS, le 15 novembre sur « Les humanités? Leur passé, leur présent, leur avenir »). 35.Il suffit d'aller à l'Université jeter un coup d'œil aux programmes des séminaires de DEA sur les panneaux d'affichage pour s'en convaincre : quels que soient les thèmes et les 
branches de philosophie abordés, les cours sont toute l'année remplacés par la venue régulière d'un conférencier extérieur, différent chaque fois, improvisant sur un sujet souvent trop vaste en 1 ou 2 heures, devant un public qu'il ne reverra jamais et qui ne le reverra jamais...

36.D'où le côté «austère » de l'amphithéâtre Paul Painlevé où est prononcée chaque soir une conférence, et d'un programme plus fondé sur le choix des sujets, que sur celui d'intervenants médiatiques (Ainsi, en philosophie, Bouveresse ( 21 janvier) plutôt que Comte-Sponville, Granger (17 janvier) plutôt que Luc Ferry, etc.)

37.I a en effet enseigné la philosophie à l'Université de Berkeley en Californie (1983), à l'Université d'Edimbourg, en Grande-Bretagne (1986) et à l'Université d'État de Sao Paulo, au Brésil (1987).

38.Cf. note 34, sur le projet de réforme de François Bayrou.

39.C'est un peu l'esprit de l'Institut Universitaire de France, qui vote des crédits alloués à la recherche pour 5 ans seulement. Y. Michaud a également fait partie du comité national du CNRS.

40.Dernier en date, par exemple, le $\mathrm{n}^{\circ} 1437 \mathrm{du}$ Point (31 mars 2000), avec en « une » : « 2250 lycées au banc d'essai. Ceux qui montent, ceux qui baissent » (cf. p. 72-74), avec un cahier détachable.

41.Créée par Larousse et Sélection du Reader's Digest en 1993, la collection «Jeunes Talents ", dirigée par Jacques Marseille, professeur à la Sorbonne, se donne pour mission de faire connaître au public les travaux de maittrise en les publiant. Le comité de sélection des ouvrages publiés est composé d'Hélène Carrère d'Encausse, Jacqueline de Romilly, Jean Bernard et Georges Duby de l'Académie Française ; de Marc Fumaroli au Collège de France, d'Alfred Grosser, professeur à l'IEP de Paris, de Michel Laclotte, présidentdirecteur du Musée du Louvre, d'Henri Miterand, professeur à l'Université Columbia de New York et d'Edgar Morin, directeur du CETSAP. Cette heureuse initiative, datée de 1993, n'eut alors qu'un faible écho dans la presse : seul Libération y consacra une page entière. On peut citer, par exemple, la maitrise de Yannick Beaubatie, Le Nihilisme et la morale de Nietzsche (Éditions Larousse et Sélection du Reader's Digest, coll. « Jeunes Talents », 1994). 42.Toujours dans le même entretien au Monde (30/01/96), intitulé « Vers la seconde révolution du livre ?", Y. Michaud se disait gêné par le fait que, lorsque l'on est homme de service public, et donc ne travaillant pas au coût réel, on puisse avoir « toute latitude pour faire des livres invendables », et il déclarait : «L'État ne doit pas couper l'herbe sous le pied des éditeurs privés. Ni servir à produire de fausses publications, actes de colloques, etc., qui sont l'occasion pour les lieux institutionnels de se faire plaisir narcissiquement... ».

43.L'apparent cynisme d'Y. Michaud sur la question du consumérisme de tout un chacun vise la mauvaise foi qui s'offusque qu'il y ait un supermarché au Louvre, alors que cela répond à une demande bien réelle des gens, puisqu'ils s'y rendent. Cela n'implique pas un jugement de valeur positif sur ce genre de choses. Il y a une différence entre décrire une réalité, décrire le réel auquel on est attentif, et y adhérer, l'approuver. Le problème, avec un philosophe au franc-parler comme Y. Michaud, est qu'on prend souvent son discours énonciatif pour une évaluation. Ce trait caractérise sa manière de philosopher comme de débattre (car, ayant remarqué l'effet produit, il en use de manière provocatrice) n'importe où, et l'amène régulièrement à de violents conflits avec des interlocuteurs qui ont cru que ce qu'il se contentait de décrire était défendu par lui...

44.On peut en effet retrouver l'UTLS sur Internet : 1) sur le site de la Mission 2000 en France (http://www.2000enfrance.com), où il y a le calendrier et le programme détaillé des 
conférences, des informations sur les conférenciers (notices biographiques, résumés de leurs conférences), et, dans une partie plus interactive, un forum de discussion avec les internautes, et la possibilité de poser des questions à certains conférenciers de l'UTLS à

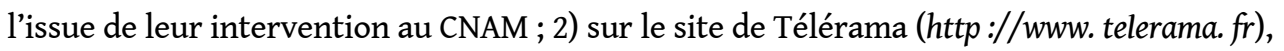
où toutes les conférences sont stockées en audio, et où vous pouvez donc les écouter et/ ou les enregistrer sur votre disque dur.

45.Il n'y a en effet pas - pour le moment - de retransmission des conférences à la radio. On trouve cependant pas mal d'interviews d'intervenants de l'UTLS : 1) sur France Culture, du lundi au vendredi, dans une émission éponyme de l'UTLS, de $11 \mathrm{~h} 30$ à $12 \mathrm{~h}$;2) sur RFI, deux fois par semaine, dans l'émission de Patrick Chompré « Le Monde Change ", de 13 h10 à $13 \mathrm{~h} 30$; 3) sur France-Info, dans les chroniques du lundi de Marie-Odile de Montchicourt, et dans son émission du samedi matin " Profession Chercheur "; 4) enfin, sur France Inter, dans l'émission de Jacques Chancel, chaque dernier dimanche du mois, « Figure de Proue ", à 17 h. Toutefois, cet été, France Culture devrait rediffuser dans leur intégralité, un choix de conférences de l'UTLS. Par ailleurs, à la télévision, on dispose des relais suivants : 1) sur LCI, tous les jeudis matin, de 9 h 40 à 10 h, Jean-François Rabilloud et Laurence Ferrari interviewent un conférencier de l'UTLS, dans leur émission « On en parle »;2) sur France 2, un dimanche sur deux, un conférencier de l'UTLS est interviewé au dernier journal $(0 \mathrm{~h} 00) ; 3)$ sur la 5 e depuis le 1er avril, 3 conférences sont rediffusées chaque semaine : à $5 \mathrm{~h}$ le samedi et dimanche matin, et à $5 \mathrm{~h} 30$ le lundi matin;3) enfin, la Chaîne Parlementaire-Assemblée Nationale, nouvellement créée, rediffuse depuis le 5 avril, l'intégralité du « feuilleton » des conférences de l'Université de Tous les Savoirs, sur Canal Satellite, TPS et le Câble.

46.Y Michaud a récemment fait effectuer par des sociologues une analyse du public de l'UTLS, à partir d'un questionnaire assez rudimentaire et " amateur ", distribué chaque jour au CNAM, et il a eu la surprise de ne pouvoir cibler précisément son public : hétérogène, composite (très jeunes ou très vieux ; pas diplômés ou surdiplômés ; venant du 3e arrondissement parisien ou bien de Bretagne ; venant chaque jour, ou bien pour une seule conférence, etc.), il demeure impossible à enfermer dans une catégorie, ce qui fait dire à Y. Michaud qu'un tel succès, qui traverse tout le corps social, traduit une véritable demande sociale, et constitue véritablement - osons le mot, pourquoi pas ? - un " phénomène de société ».

47.Ainsi, par exemple, Yannick Maignien, en poste à l'Ambassade de France au Mexique, s'est vu chargé de la Mission de réussir à diffuser les conférences de l'UTLS sur les réseaux universitaires mexicains, dans le cadre d'un projet intitulé « Université virtuelle EuroAmerica ", au Centre de Ressources Casa de Francia (http://www. francia.org.mx), et a sollicité l'UTLS pour y parvenir.

48.La Columbia University (6 Prix Nobel, 31 autres prix), la London School of Economics and Political Science (28 chefs d'État y ont étudié ou enseigné), la Cambridge University, la Smithsonian Institution's National Museum of Natural History (Musée le plus visité au monde), la British Library (qui possède une des plus importantes collections du patrimoine mondial), la New York Public Library et les Cambridge University Press (plus vieilles éditions du monde, qui ont numérisé plus de 600000 images) se sont associés dans un consortium d'excellence scientifique sur le site de "fathom. com », sur lequel ils proposent toute une série d'offres variées dans le champ du savoir : interviews, articles, conférences, forums de discussion, produits et services culturels divers (livres, CDRom, voyages, etc.), le tout en relation constante avec les chercheurs les plus éminents, et les meilleurs spécialistes dans leurs domaines respectifs, qui peuvent aller de la médecine à l'informatique, en 
passant par l'histoire de l'art, le droit, etc. Premier site de savoir interactif, « fathom. com » s'adresse aux amoureux du savoir comme aux visiteurs ponctuels à la recherche d'informations précises et fiables.

49.«Changez tout, mais surtout ne touchez à rien », disait Bruno Frappat, dans un éditorial du Monde de l'Éducation, il y a quelques années, pour résumer l'opinion des Français sur l'Éducation Nationale !

50.Nietzsche, dans la Généalogie de la morale (1887, cf. Euvres Complètes aux Éditions Gallimard, 1971, tome VII, p. 213-347), décèle ce subterfuge à partir d'analyses philologiques. Sous une apparente constance des termes « bon et mauvais » dans le langage, se cache en réalité une lutte de pouvoir entre deux forces dont la seconde a emprunté le masque de la 1re pour l'abattre, et jusqu'à son vocabulaire. Pour schématiser une généalogie bien compliquée : au départ était la caste des guerriers, ces êtres forts et sûrs d'eux, qui conquéraient tout sur leur passage ; seuls au monde, ils désignaient les choses pour la première fois, et organisaient ainsi leur Weltanschauung en nommant « bon ", ce qui leur apportait surcroît de force, et « mauvais », le reste. Vint ensuite la caste des prêtres. Jaloux des guerriers et de leur force, ils ne leur ressemblaient en aucune façon et avaient des valeurs tout à fait opposées : haine de la violence, haine du bonheur, haine en général d'ailleurs. Mais, pour vaincre, ils empruntèrent le masque des guerriers : sous les mêmes mots, « bon et méchant ", ils édifièrent un système de valeurs contraires, et prirent le masque d'un merveilleux « amour » de l'humanité, ressemblant à la « générosité » des guerriers, pour dissimuler leur haine et leur ressentiment. Pour Nietzsche, ces prêtres - terme sous lequel il entend l'Église Catholique - triomphèrent ainsi, en empruntant le masque des valeurs - et jusqu'aux mots les désignant - de la force qu'ils voulaient vaincre : les forts, les puissants, les sûrs d'eux, les violents, les heureux (cf. Première Dissertation : « Bien et mal », « Bon et mauvais »).

51.Cf. l'ouvrage que Deleuze consacra à Nietzsche: Nietzsche et la Philosophie (1962, PUF, réédition en 1991, dans la coll. «Bibliothèque de philosophie contemporaine »).

52.Dont la tradition de la «frontier », à l'origine de leur naissance, les pousse évidemment à rechercher et à accepter le nouveau avec engouement. Tout discours politique qui se veut persuasif aux États-Unis se doit de se référer à l'esprit pionnier des Américains, et à leur proposer une nouvelle " conquête de l'Ouest ", une nouvelle " frontière » à dépasser. Comme chacun sait, Kennedy ne s'y est pas trompé dans son fameux discours sur la nouvelle « frontière » dans les années 1960.

53.Dessinateur au Monde, qui a illustré bien des projets de la Mission 2000, dont l'UTLS. 54.Comme on peut le constater, Yves Michaud ne dit rien de vraiment neuf sur Internet, et l'état des savoirs sur Internet. Mais, là où d'autres intellectuels sautent sans résister à la conclusion tranchée selon laquelle Internet est « une poubelle » (par exemple, Umberto Eco, dans « À toutes fins utiles », dans Entretiens sur la fin des temps, Fayard, 1998, p.

235-295), Yves Michaud adopte un point de vue plus nuancé, fondé sur l'observation de ce qui se passe vraiment : la reconstitution spontanée et « horizontale » d'instances évaluatives des savoirs sur le net.

55.Telle que la pratiquent ou veulent la pratiquer, par exemple, « Diffusion Contrôle » ( http://www.mediametrie.fr), ou l'ADBS (Association des Professionnels de l'information et de la Documentation), bien que l'État travaille à une législation en la matière (cf. section du rapport et des études Internet et les réseaux numériques, étude adoptée par l'Assemblée générale du Conseil d'État le 2 juillet 1998, disponible en ligne : http:// www.internet.gouv.fr/francais/textesref/rapce98/rap4.html). 
56. C'est l'idée des philosophes du XVIII ${ }^{\mathrm{e}}$ siècle, Voltaire, Rousseau, mais aussi Kant, auteur du célèbre opuscule : Qu'est-ce que s'orienter dans la pensée?, aux fondements des

entreprises de vulgarisation, de l'Encyclopédie, de la Révolution française, et de la III ${ }^{\mathrm{e}}$ République.

57.C'est d'ailleurs la «leçon » qui a été tirée de la soudaine baisse de fréquentation (tout est relatif : on a cessé de refouler des gens : il n'y avait plus que 500 personnes au lieu de 700 ou 800 !) qui a été observée durant la pause épistémologique de l'UTLS (c'est-à-dire de réflexion sur le savoir) à la mi-janvier (du 14 au 22 janvier) : les gens viennent volontiers pour qu'on leur donne du savoir, des données concrètes, mais préfèrent se faire leur opinion eux-mêmes, réfléchir eux-mêmes, sur ce savoir. Où l'on voit que l'« enseignement » marche dans les deux sens : l'UTLS est une série de « leçons » prodiguées aux gens ; en retour, elle permet d'apprécier, sur un plan sociologique, toute une série de variables précieuses les concernant, de découvrir le social sous un nouvel angle.

58. Cette belle idée des Lumières s'est abâtardie sous la III République, exaspérée qu'elle fut : c'est elle qui imprégna les entreprises de vulgarisation d'alors, mais tout autant la politique impérialiste et colonialiste de la France. Cette conception dramatique et finalement exagérée du savoir comme source de «salut » doit donc être nuancée, surtout

à la fin du $\mathrm{XX}^{\mathrm{e}}$ siècle, où toutes les donnes sont changées.

59.Y. Michaud formule ici deux idées qui lui sont chères en une : 1) Les gens ne sont pas si bêtes que cela, en savent toujours suffisamment et réfléchissent toujours suffisamment pour se débrouiller dans la vie de tous les jours. Loin d'être les individus ignares et passifs qu'ont souvent décrits les sociologues, les gens en savent pas mal, et beaucoup sur euxmêmes, grâce au savoir sur eux que leur renvoient les moyens de communications, même si leur savoir n'est pas très organisé. Le problème, c'est que nous vivons à une époque où les gens et les sociétés n'ont jamais été aussi savants sur eux-mêmes, mais, précisément, si l' organisation de ces connaissances est en reste, c'est parce qu'aucune « autorité » ne subsiste sur laquelle les appuyer (cf. un auteur de prédilection d'Y. Michaud: Hans Magnus Enzensbgerger, « De l'ignorance », dans Médiocrité et Folie, Gallimard, coll. « Le Messager », 1991, p. 23-36 ; et Y. Michaud lui-même, La Violence apprivoisée, 1996, Hachette, coll. «Questions de société », p. 86-87) ; 2) Loin d'être bêtes, les gens réfléchissent tellement et sans cesse à leurs conduites qu'ils en changent, une fois qu'ils ont pris conscience de leurs réflexions ou de celles d'autres sur eux (journaux, presse) : c'est le phénomène de « réflexivité », concept inventé par le sociologue Anthony Giddens (auteur cher à Y. Michaud également, et qui viendra peut-être clore l'UTLS le 31 décembre en nous parlant de cette notion complexe). « La réflexivité de la vie moderne, c'est l'examen et la révision constants des pratiques sociales, à la lumière des informations concernant ces pratiques mêmes, ce qui altère constitutivement leur caractère » (A. Giddens, Les Conséquences de la modernité, L'Harmattan, coll. « Théorie sociale contemporaine », 1994, p. 45). "La même société qui ne cesse de connaître (...) ne cesse ainsi de se connaître elle-même ", grâce à ce flux continu d'informations qui est véhiculé par les media et qui fournit des « représentations de la société et des individus à eux-mêmes ». En outre, la société et les individus réagissent en retour à cette auto-objectivation en changeant leurs comportements, d'où d'autres représentations ensuite en circulation, entraînant d'autres réajustements, etc. : le cycle est infini. (Cf. Y. Michaud, La Violence apprivoisée, p. 82 et 84 ). 
60.Étant donné ce qui précède, Y. Michaud réfute absolument la thèse bourdieusienne d'un rôle des intellectuels à jouer dans le " guidage » des masses, et du devoir de ceux-ci de parler en leur nom. Les gens sont suffisamment intelligents pour comprendre, réfléchir et agir par eux-mêmes ; loin de constituer une masse inerte qui serait passivement manipulée par le pouvoir politique ou autre, ils ne cessent de contremanipuler, et de bouger, telle une " cible mouvante » (expression du spécialiste des sciences sociales canadien, Ian Hacking, auteur de L'Âme réécrite. Étude sur la personnalité multiple et les sciences de la mémoire, Éditions de l'Institut Synthélabo, coll. « Les empêcheurs de penser en rond », 1998, 451 pages) grâce, précisément, à la " réflexivité ». Quelqu'un comme Olivier Mongin, directeur de la revue Esprit et auteur de nombreuses études sur les intellectuels (comme Face au scepticisme. Les mutations du paysage intellectuel, 1994, La Découverte, réédition chez Hachette, coll. « Pluriel », en 1998), a lui aussi pris en considération cette nouvelle donne, qui amène à revoir le rôle des intellectuels à la baisse, mais que, pour sa part, il ne va pourtant pas jusqu'à éliminer... (cf. les deux dernières livraisons d'Esprit, « Splendeurs et Misères de la vie intellectuelle, I, II, et III », n 3-4 de mars-avril 2000 , et $n^{\circ} 5$ de mai 2000 ; et en particulier son article introductif au n ${ }^{\circ} 3-4:$ « Splendeurs et Misères de la vie intellectuelle (I), Fin de partie ?»).

61.La «Semeuse » est dessinée par Eugène Grasset en 1890, et complétée par la devise : « Je sème à tous vents ». Auparavant, en 1876, le premier logo de Larousse représentait une dent-de-lion (nom usuel du pissenlit à cause de la découpure de ses feuilles) dont les graines s'éparpillent au gré du vent et ornée de la devise due à Émile Reiber, architecte et décorateur français (1826-1893) : «Je sème à tout vent ». L'idée de semence, de germe, de fructification par l'instruction, allusion transparente à la parabole de l'Évangile, se trouve déjà dans les œuvres de Pierre Larousse, notamment dans la préface de son Grand Dictionnaire. Modifiée plusieurs fois dans son design, la Semeuse d'aujourd'hui, au graphisme épuré, est le fruit d'un travail collectif à partir du dessin de Grasset, auquel a participé Yann Penor's en 1993. Sa célébrité est telle que les studios Disney lui ont adressé un clin d'œil dans le dessin animé La Belle et la Bête...

\section{AUTEUR}

\section{YVES MICHAUD}

Professeur de philosophie à Paris I, concepteur de l'Université de Tous les Savoirs, organisée par la Mission 2000 en France. 